\title{
Prediction of Microelement Fertilizers for Wheat Yield Optimizing with GMDH-type Neural Network
}

\author{
M. J. Seghatoleslami, G.R. Mousavi, N. Beheshti Nezhad, H. Beheshti Nezhad \\ Islamic Azad University, Birjand Branch, Iran \\ O. Parsapour
}

Khouzestan Ramin Agriculture and Natural Resources University, Iran

\begin{abstract}
Wheat is the main carbohydrate source which is taken to be human's food too. Considering the irregular population augmentation in the world the provision of food stuffs is a predicament of intense significance. Prediction of wheat yield using different quantity microelements is an important issue as a key parameter in increase of wheat production .Group Method of Data Handing (GMDH) algorithm is self-organizing approach by which gradually complicated models are generated based on the evaluation of their performances on asset of multi-input-single-output data pairs .The GMDH was firstly developed by Ivakhenko as a multivariate analysis method for complex system modeling and identification. In this way, GMDH was used to circumvent the difficulty of knowing prior knowledge of mathematical model of the process being considered .The main idea of GMDH is to build an analytical function in a feed forward network based on a quadratic node transfer function whose coefficient are obtained using regression technique. In fact, real GMDH algorithm in which model coefficient are estimated by means of the least squares method has been classified in two complete induction and in complete induction, which represent the combinational and multi layered iterative algorithms, respectively.
\end{abstract}

Keywords: wheat yield, microelement, neural network, GMDH.

\section{Introduction}

Wheat is the most strategic crop in world. It is the main staple crop and supplies more than $45 \%$ of the protein and 55\% of the caloric requirement in human. Despite a domestic production of 11 million tons a year, Iran is an importer of wheat (S. Ghorbani, B.N. Khiabani, I. Amini and M.R. Ardakani, 2009).

Balanced fertilization and micronutrients reported as becoming deficient worldwide, zinc (Zn) and iron (Fe) are the most important elements (P. Lucca, F. Hurrel, I. Potrykus, 2001). Zinc is an essential micronutrient for plants and animals and there is evidence of deficiency (B. J. Alloway, 2004). Zinc plays a major role in wheat production (Z. Regal, R.D Graham, 1995).

In this study, these models were applied to forecast the production of wheat crop in Birjand. In recent

M. J. Seghatoleslami, Associate Professor of Islamic Azad University, Birjand Branch, Iran. Email: mjseghat@yahoo.com (mjseghat@iaubir.ac.ir).

G.R. Mousavi, Assistant Professor of Islamic Azad University, Birjand Branch, Iran. Email: s_reza1350@yahoo.com.

N. Beheshti Nezhad, Former M.Sc Student of Islamic Azad University, Birjand Branch, Iran.

H. Beheshti Nezhad, Faculty Member of Islamic Azad University, Birjand Branch, Iran. Email: civileng_78@yahoo.com.

O. Parsapour, Ph.D. Student of Agronomy, Khouzestan Ramin Agriculture and Natural Resources University, Iran. 
years experience has been gained in using neural networks for predicting crop yields (T. Heinzow, R.S.J. Tol, 2003).

One of important causes for increase of wheat yield is use of optimized microelement fertilizers. Prediction of wheat yield is an important issue as a key parameter in this optimization.

System identification techniques are applied in many fields in order to model and predict the behaviors of unknown and/or very complex systems based on given input-output data (K. J. Astrom, P. Eykhoff, 1971). Theoretically, in order to model a system, it is required to understand the explicit mathematical input-output relationship precisely. Such explicit mathematical modeling is, however, very difficult and is not readily tractable in poorly understood systems. Alternatively, soft-computing methods (E. Sanchez, T. Shibata, L.A. Zadeh, 1997), which concern computation in imprecise environment, have gained significant attention. The main components of soft computing, namely, fuzzy-logic, neural network, and genetic algorithm have shown great ability in solving complex non-linear system identification and control problems. Several research efforts have been expended to use evolutionary methods as effective tools for system identification (E. Sanchez, T. Shibata, L.A. Zadeh, 1997) (H. Iba, H. deGaris, T. Sato, 1996). Among these methodologies, Group Method of Data Handling (GMDH) algorithm is self-organizing approach by which gradually complicated models are generated based on the evaluation of their performances on a set of multi-input-single-output data pairs ( $i=1$, $2, \ldots, \mathrm{M})$. The GMDH was firstly developed by Ivakhnenko (A.G.Ivakhnenko, 1971) as a multivariate analysis method for complex systems modeling and identification. In this way, GMDH was used to circumvent the difficulty of knowing a priori knowledge of mathematical model of the process being considered. In other words, GMDH can be used to model complex systems without having specific knowledge of the systems. The main idea of GMDH is to build an analytical function in a feed forward network based on a quadratic node transfer function (S. J. Farlow, 1984) whose coefficients are obtained using regression technique. In fact, real GMDH algorithm in which model coefficients are estimated by means of the least squares method, has been classified into complete induction and incomplete induction, which represent the combinatorial (COMBI) and multilayered iterative algorithms (MIA), respectively (S. J. Farlow, 1984). In recent years, however, the use of such self-organizing network leads to successful application of the GMDH-type algorithm in a broad range area in engineering, science, and economics (S. J. Farlow, 1984) (N. Nariman-Zadeh, A. Darvizeh, R. Ahmad-Zadeh, 2003).

In this paper, wheat yield is modeled and predicted using GMDH-type neural networks based on some experimental data. The aim of such modeling is to show how wheat yield change with the variation of levels of fertilizers (Zinc \& Iron). In this way, genetic algorithms are deployed in a new approach to design the whole architecture of the GMDH-type neural networks, i.e., the number of neurons in each hidden layer and their connectivity configuration, in combination with using regression method to find optimal set of appropriate coefficients of quadratic expressions for modeling and prediction of wheat yield. The connectivity configuration is not limited to the adjacent layers, as have already proposed in (N. Nariman-Zadeh, A. Darvizeh, R. Ahmad-Zadeh, 2003), so that general structure GMDH-type neural networks, in which neurons in any layers can be connected to each other, are evolved. Furthermore, a new yet simple encoding scheme has been introduced to present the genotype of general structure GMDH-type neural networks so that the length of chromosome is kept minimal and thus leads to simpler evolutionary operations including crossover and mutation. Such encoding scheme must allow for producing and/or switching both general and simplified networks using the genetic operators during evolutionary process. 


\section{Modeling Using GMDH_ Type Neural Networks}

By means of GMDH algorithm a model can be represented as set of neurons in which different pairs of them in each layer are connected through a quadratic polynomial and thus produce new neurons in the next layer. Such representation can be used in modeling to map inputs to outputs. The formal definition of the identification problem is to find a function $\hat{f}$ so that can be approximately used instead of actual one, $f$, in order to predict output $\hat{\mathrm{y}}$ for a given input vector $\mathrm{X}=(x 1, x 2, x 3, \ldots, x n)$ as close as possible to its actual output $\mathrm{y}$. Therefore, given $\mathrm{M}$ observation of multi-input-single-output data pairs so that

$$
y_{i}=f\left(x_{i 1}, x_{i 2}, x_{i 3}, \ldots, x_{i n}\right) \quad(i=1,2, \ldots, M)
$$

It is now possible to train a GMDH-type neural network to predict the output values ŷi for any given input vector $\mathrm{X}=\left(x_{i 1}, x_{i 2}, x_{i 3}, \ldots, x_{i n}\right)$, that is

$$
\hat{y}_{i}=\hat{f}\left(x_{i 1}, x_{i 2}, x_{i 3}, \ldots, x_{i n}\right) \quad(i=1,2, \ldots, M)
$$

The problem is now to determine a GMDH-type neural network so that the square of difference between the actual output and the predicted one is minimized, that is

$$
\sum_{i=1}^{M}\left[\hat{f}\left(x_{i 1}, x_{i 2}, x_{i 3}, \ldots, x_{i n}\right)-y_{i}\right]^{2} \rightarrow \min
$$

General connection between inputs and output variables can be expressed by a complicated discrete form of the Volterra functional series in the form of

$$
y=a_{0}+\sum_{i=1}^{n} a_{i} x_{i}+\sum_{i=1}^{n} \sum_{j=1}^{n} a_{i j} x_{i} x_{j}+\sum_{i=1}^{n} \sum_{j=1}^{n} \sum_{k=1}^{n} a_{i j k} x_{i} x_{j} x_{k}+\ldots
$$

which is known as the Kolmogorov-Gabor polynomial (S. J. Farlow, 1984) (N. Nariman-Zadeh, A. Darvizeh, R. Ahmad-Zadeh, 2003).This full form of mathematical description can be represented by a system of partial quadratic polynomials consisting of only two variables (neurons) in the form of

$$
\hat{y}=G\left(x_{i}, x_{j}\right)=a_{0}+a_{1} x_{i}+a_{2} x_{j}+a_{3} x_{i} x_{j}+a_{4} x_{i}^{2}+a_{5} x_{j}^{2}
$$

In this way, such partial quadratic description is recursively used in a network of connected neurons to build the general mathematical relation of inputs and output variables given in equation 4 . The coefficient $a_{i}$ in equation 5 is calculated using regression techniques (S. J. Farlow, 1984) (N. Nariman-Zadeh, A. Darvizeh, R. Ahmad-Zadeh, 2003). So that the difference between actual output, $y$, and the calculated one, $\hat{y}$, for each pair of $x_{i}, x_{j}$ as input variables is minimized. Indeed, it can be seen that a tree of polynomials is constructed using the quadratic form given in equation 5 whose coefficients are obtained in a least-squares sense. In this way, the coefficients of each quadratic function $G_{i}$ are obtained to optimally fit the output in the whole set of input-output data pair, that is

$$
E=\frac{\sum_{i=1}^{M}\left(y_{i}-G_{i}\right)^{2}}{M} \rightarrow \min
$$

In the basic form of the GMDH algorithm, all the possibilities of two independent variables out of total $n$ input variables are taken in order to construct the regression polynomial in the form of equation 5 that best fits the dependent observations $\left(y_{i}, i=1,2, \ldots, M\right)$ in a least-squares sense. Consequently $(n-1) / 2$ neurons will be built up in the first hidden layer of the feed forward network from the observations $\left\{\left(y_{i}, x_{i p}, x_{i q}\right),(i=1,2, \ldots\right.$, M) $\}$ for different $p, q \in\{1,2, \ldots, n\}$. In other words, it is now possible to construct M data triples $\left\{\left(y_{i}, x_{i p}, x_{i q}\right)\right.$; 
$(i=1,2, \ldots, M)\}$ from observation using such $p, q \in\{1,2, \ldots, n\}$ in the form

$$
\left[\begin{array}{cc:c}
x_{1 p} & x_{1 q} & y_{1} \\
x_{2 p} & x_{2 q} & y_{2} \\
\hdashline X_{M p} & x_{M q} & y_{M}
\end{array}\right]
$$

Using the quadratic sub-expression in the form of equation 5 for each row of $\mathrm{M}$ data triples, the following matrix equation can be readily obtained as

$$
\mathrm{Aa}=\mathrm{Y}
$$

where a is the vector of unknown coefficients of the quadratic polynomial in equation 5

$$
\mathrm{a}=\left\{\mathrm{a}_{0}, \mathrm{a}_{1}, \mathrm{a}_{2}, \mathrm{a}_{3}, \mathrm{a}_{4}, \mathrm{a}_{5}\right\}
$$

and

$$
\mathrm{Y}=\left\{y_{1}, y_{2}, y_{3}, \ldots, y_{\mathrm{n}}\right\} \mathrm{T}
$$

is the vector of output's value from observation. It can be readily seen that

$$
A=\left[\begin{array}{cccccc}
1 & x_{1 p} & x_{1 q} & x_{1 p} x_{1 q} & x_{1 p}^{2} & x_{1 q}^{2} \\
1 & x_{2 p} & x_{2 q} & x_{2 p} x_{2 q} & x_{2 p}^{2} & x_{2 q}^{2} \\
\hdashline 1 & x_{M p} & x_{M q} & x_{M p} x_{M q} & x_{M p}^{2} & x_{M q}^{2}
\end{array}\right]
$$

The least-squares technique from multiple-regression analysis leads to the solution of the normal equations in the form of

$$
a=\left(A^{T} A\right)^{-1} A^{T} Y
$$

which determines the vector of the best coefficients of the quadratic equation 5 for the whole set of $M$ data triples. It should be noted that this procedure is repeated for each neuron of the next hidden layer according to the connectivity topology of the network.

However, such a solution directly from normal equations is rather susceptible to round off errors and, more importantly, to the singularity of these equations.

\section{Application of SVD to the Design of GMDH-Type Neural Networks}

The SVD of a matrix, $A \in \mathfrak{R} M \times 6$ is a factorization of the matrix into the product of three matrices, column-orthogonal matrix, $U \in \mathfrak{R}^{M \times 6}$ diagonal matrix $W \in \mathfrak{R}^{6 \times 6}$ with non-negative elements (singular values), and orthogonal matrix $V \in \mathfrak{R}^{6 \times 6}$ such that

$$
\mathrm{A}=\mathrm{UWV}^{\mathrm{T}}
$$

The problem of optimal selection of vector of the coefficients in equations 7,11 is firstly reduced to finding the modified inversion of diagonal matrix W (W.H. Press, S.A. Teukolsky, W.T. Vetterling, B.P. Flannery, 1992) in which the reciprocals of zero or near zero singulars (according to a threshold) are set to zero. Then, such optimal a is calculated using the following relation

$$
\mathrm{a}=\mathrm{V}[\operatorname{diag}(1 / \mathrm{wj})] \mathrm{U}^{\mathrm{T}} \mathrm{Y}
$$

Such parametric identification problem is part of the general problem of modeling when structure identification is considered together with the parametric identification problem simultaneously. In this work, an encoding scheme developed by authors (N. Nariman-zadeh, A. Darvizeh, A. Jamali, A. Moeini, 2005) is used in an evolutionary approach for simultaneous determination of structure and parametric identification of GMDH neural networks. 


\section{Application of Gas in the Topology Design of GMDH}

Evolutionary methods such as genetic algorithms have been widely used in different aspects of design in neural networks because of their unique capabilities of finding a global optimum in highly multi-modal and/or non-differentiable search space (H. Iba, H. deGaris, T. Sato, 1996) (N. Nariman-zadeh, A. Darvizeh, A. Jamali, A. Moeini, 2005). Such stochastic methods are commonly used in the training of neural networks in terms of associated weights or coefficients which have successfully performed better than traditional gradient-based techniques (N. Nariman-Zadeh, A. Darvizeh, R. Ahmad-Zadeh, 2003). The literature shows that a wide range of evolutionary design approaches either for architectures or for connection weights separately, in addition to efforts for them simultaneously (X. Yao, 1999). In the most GMDH-type neural network, neurons in each layer are only connected to neuron in its adjacent layer as it was the case in Methods I and II previously reported in (N. Nariman-Zadeh, A. Darvizeh, R. Ahmad-Zadeh, 2003). Taking this advantage, it was possible to present a simple encoding scheme for the genotype of each individual in the population as already proposed by authors (N. Nariman-Zadeh, A. Darvizeh, R. Ahmad-Zadeh, 2003).

\section{The Genome Representation of Generalized GMDH Neural Networks}

In the generalized GMDH neural networks, neurons connections can occur between different layers which are not necessarily very adjacent ones, unlike the conventional structure GMDH neural networks in which such connections only occur between adjacent layers. For example, a network structure which depicted in Figure 1. shows such connection of neuron ad directly to the output layer. Consequently, this generalization of network's structure can evidently extend the performance of generalized GMDH neural networks in modeling of real-world complex processes. Such generalization is accomplished by repeating the name of the neuron which directly passing the next layers. In Figure 1., neuron ad in the first hidden layer is connected to the output layer by directly going through the second hidden layer. Therefore, it is now very easy to notice that the name of output neuron (network's output) includes ad twice as abbcadad. In other words, a virtual neuron named adad has been constructed in the second hidden layer and used with abbc in the same layer to make the output neuron abbcadad as shown in the Figure 1. It should be noted that such repetition occurs whenever a neuron passes some adjacent hidden layers and connects to another neuron in the next 2nd, or 3rd, or 4th, or ... following hidden layer. In this encoding scheme, the number of repetition of that neuron depends on the number of passed hidden layers, ñ, and is calculated as. It is easy to realize that a chromosome such as abab bcbc, unlike chromosome abab acbc for example, is not a valid one in GS-GMDH networks and has to be simply re-written as abbc .

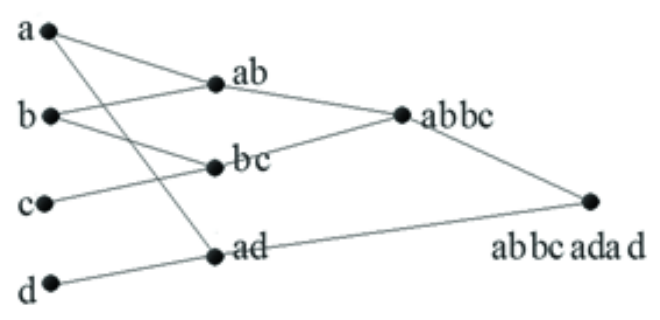

Figure 1. A Generalized GMDH network structure of a chromosome.

\section{Genetic Operator for GMDH Network Reproduction}

The genetic operators of crossover and mutation can now be implemented to produce two off springs from two parents. The natural roulette wheel selection method is used for choosing two parents producing two off 
springs. The crossover operator for two selected individuals is simply accomplished by exchanging the tails of two chromosomes from a randomly chosen point as shown in Figure 2. It should be noted, however, such a point could only be chosen randomly from the set $\left\{2^{1}, 2^{2}, \ldots, 2^{n^{+1}}{ }_{1}\right\}$ where $n_{l}$ is the number of hidden layers of the chromosome with the smaller length. It is very evident from Figures 2, 3. that the crossover operation can certainly exchange the building blocks information of such generalized GMDH neural networks so that the two types of generalized GMDH and conventional GMDH-type neural networks can be converted to each other, as can be seen from Figure 3. In addition, such crossover operation can also produce different length of chromosomes which in turn leads to different size of either generalized GMDH or conventional GMDH network structures. Similarly, the mutation operation can contribute effectively to the diversity of the population. This operation is simply accomplished by changing one or more symbolic digits as genes in a chromosome to another possible symbol, for example, abbcadad to abbccdad. It is very evident that mutation operation can also convert a generalized GMDH network to a conventional GMDH network or vice versa. It should be noted that such evolutionary operations are acceptable provided a valid chromosome is produced .Otherwise; these operations are simply repeated until a valid chromosome is constructed.

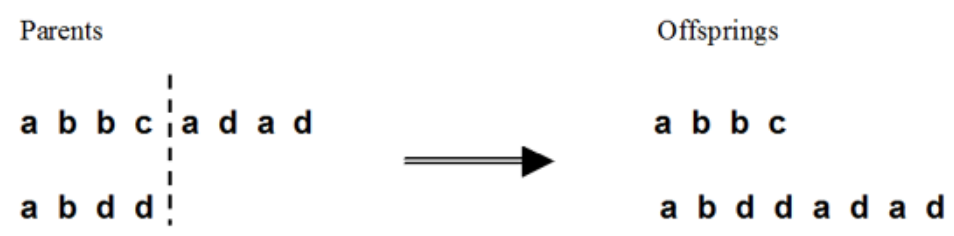

Figure 2. Crossover operation for two individuals in GS-GMDH networks.

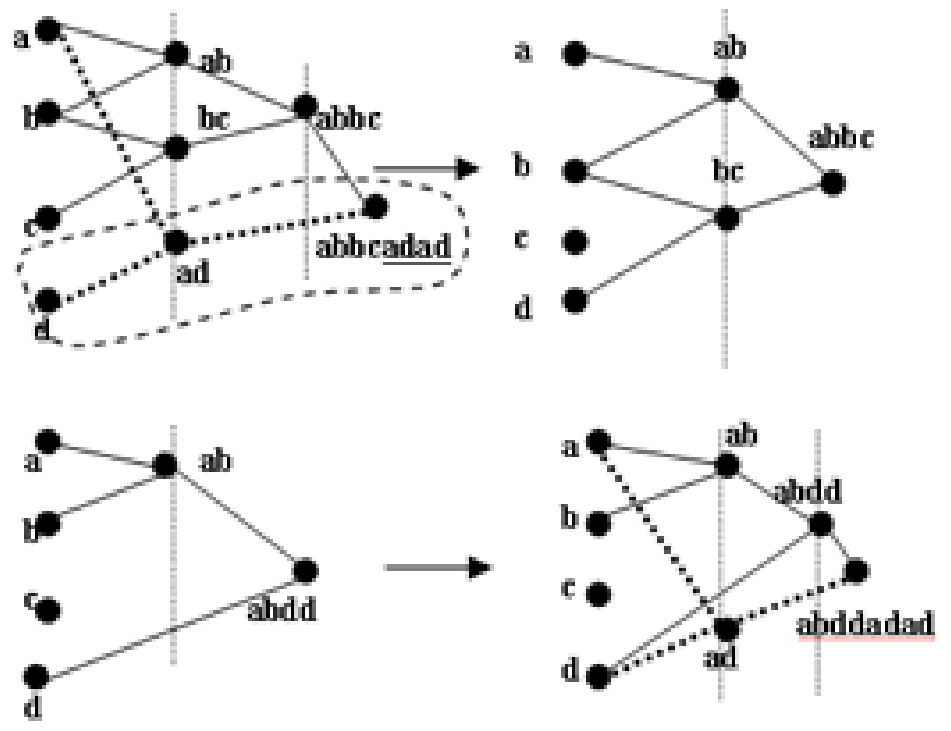

Figure 3. Crossover operation on two GS-GMDH networks

The incorporation of genetic algorithm into the design of such GMDH-type neural networks starts by representing each network as a string of concatenated sub-strings of alphabetical digits. The fitness, $(\Phi)$ of each entire string of symbolic digits which represents a GMDH-type neural network to model wheat yield is evaluated in the form

$$
\Phi=1 / \mathrm{E}
$$


where $\mathrm{E}$, is the mean square of error given by equation 6 , is minimized through the evolutionary process by maximizing the fitness $\Phi$. The evolutionary process starts by randomly generating an initial population of symbolic strings each as a candidate solution. Then, using the aforementioned genetic operations of roulette wheel selection, crossover, and mutation, the entire populations of symbolic strings improve gradually. In this way, GMDH-type neural network models of wheat yield with progressively increasing fitness, $\Phi$, are produced until no further significant improvement is achievable.

\section{Materials and Method}

This research was conducted in the 2008-2009 growing season at the Agricultural Research Center of Islamic Azad University, Birjand branch, Iran. Longitude, latitude and altitude of Birjand are 59 $13^{\prime}$, 32 $2^{\circ} 53^{\prime}$ and $1470 \mathrm{~m}$, respectively. Some physicochemical properties of experimental soil have been shown in Table 1 .

Table 1

Characteristics of Field Soil Sample before Planting.

\begin{tabular}{llllllllllll}
\hline $\begin{array}{l}\mathrm{n} \\
(\mathrm{mg} / \mathrm{kg})\end{array}$ & $\begin{array}{l}\mathrm{Cu} \\
(\mathrm{mg} / \mathrm{kg})\end{array}$ & $\begin{array}{l}\mathrm{Zn} \\
(\mathrm{mg} / \mathrm{kg})\end{array}$ & $\begin{array}{l}\mathrm{Fe} \\
(\mathrm{mg} / \mathrm{kg})\end{array}$ & $\begin{array}{l}\mathrm{K} \\
\text { p.p.m }\end{array}$ & $\begin{array}{l}\text { P.p.m } \\
\%\end{array}$ & $\begin{array}{l}\mathrm{N} \\
\%\end{array}$ & $\begin{array}{l}\text { OC } \\
\%\end{array}$ & EC & Text & $\begin{array}{l}\text { Depth } \\
(\mathrm{cm})\end{array}$ \\
\hline 6.87 & 0.34 & 0.22 & 4.62 & 235 & 5.9 & 0.0037 & 0.04 & 8.15 & 9.8 & Sandy-Lume & $0-30$ \\
\hline
\end{tabular}

The experiment was conducted as factorial based on completely randomized block design with 3 replications. Factors were included Fe and Zn fertilizers ( $0 \%, 0.2 \%$ and $0.5 \%$ for $\mathrm{Zn}$ and $0 \%, 0.5 \%, 0.7 \%$ and $1 \%$ for Fe). The used cultivar of wheat was Bami.

Mentioned levels of Fe and $\mathrm{Zn}$ used as iron sulfate and zinc sulfate. The fertilizers sprayed on leaves two times at the growth period of wheat. Seed yield determined after harvesting. The yield indicated in Table 2. MATLAB software was used for programming and modeling of wheat yield.

Table 2

Grain yield in wheat $\left(\mathrm{g}^{-2}\right)$ Using Variable Zn And Fe Rates

\begin{tabular}{lllll}
\hline $\begin{array}{l}\mathrm{ZnSo}_{4} \\
\%\end{array}$ & $\begin{array}{l}\mathrm{FeSO}_{4} \\
\%\end{array}$ & $\begin{array}{l}\text { bolck1 } \\
\text { Grain yield }\left(\mathrm{g}^{-2}\right)\end{array}$ & $\begin{array}{l}\text { block2 } \\
\text { Grain yield } \\
\left(\mathrm{gg}^{-2}\right)\end{array}$ & $\begin{array}{l}\text { block3 } \\
\text { Grain yield } \\
\left(\mathrm{g}^{-2}\right)\end{array}$ \\
\hline 0 & 0 & 793.9 & 1013.2 & 841.7 \\
0 & 0.5 & 824.4 & 819.4 & 681.8 \\
0 & 0.7 & 1008.4 & 628.5 & 640.8 \\
0 & 1 & 809.9 & 870.2 & 957 \\
0.2 & 0 & 962.8 & 941 & 892.7 \\
0.2 & 0.5 & 1153.3 & 950.9 & 732.3 \\
0.2 & 0.7 & 962.6 & 749.5 & 878 \\
0.2 & 1 & 1041 & 772.4 & 644.9 \\
0.5 & 0 & 747.9 & 900.6 & 773 \\
0.5 & 0.5 & 748.9 & 929.7 & 800.9 \\
0.5 & 0.7 & 796.4 & 907.7 & 1025 \\
0.5 & 1 & 758.8 & 906.6 & 542.6 \\
\hline
\end{tabular}

\section{Results and Discussion}

There have been a total number of 12 input-output experimental data considering levels of zinc (\%) and iron (\%) as input and wheat yield as output. The GMDH-type neural networks are now used for such 
input-output data to find the polynomial model of wheat yield in respect to their effective input parameters. In order to genetically design such GMDH-type neural network described in previous section, a population of 50 individuals with a crossover probability of 0.7 and mutation probability of 0.07 has been used in 300 generation which no further improvement has been achieved for such population size. The structure of the evolved 2-hidden layer GMDH-type neural network is shown in Figure 4. The very good behavior of such GMDH-type neural network models are also depicted in Figure 5. The corresponding polynomial representation of such model is:

$$
\begin{array}{rr}
\mathrm{Y} 1=781.89603384+384.825626305(\mathrm{Zn})+317.952595615(\mathrm{Fe})-527.88888900(\mathrm{Zn})^{2}-259.509489953(\mathrm{Fe})^{2} \\
-97.874875918(\mathrm{Zn})(\mathrm{Fe}) & (15 \mathrm{a}) \\
\mathrm{Y} 2=-0.03117058+0.989129533(\mathrm{Y} 1)+0.086687142(\mathrm{Fe})+0.000013295(\mathrm{Y} 1)^{2}+2.905766169(\mathrm{Fe})^{2}- \\
0.003948248(\mathrm{Y} 1)(\mathrm{Fe}) & (15 \mathrm{~b}) \\
\mathrm{Y} 3=-0.063954829+0.998469086(\mathrm{Y} 2)-0.112658466(\mathrm{Zn})+0.00000212(\mathrm{Y} 2)^{2}+3.114825510(\mathrm{Zn})^{2} \\
-0.002383419(\mathrm{Y} 2)(\mathrm{Zn}) & (15 \mathrm{c})
\end{array}
$$

However, in order to demonstrate the prediction ability of evolved GMDH-type neural networks, the data has been divided into two different sets, namely, training and testing sets. The training set, which consists of 10 out of 12 inputs-output data pairs, is used for training the neural network models the evolutionary method of this paper. The testing set, which consists of 2 unforeseen inputs-output data samples during the training process, is merely used for testing to show the prediction ability of such evolved GMDH-type neural network models during the training process. The structure of the evolved 2-hidden layer GMDH-type neural network is that same as Figure 4. The very good behavior of such GMDH-type neural network models are also depicted in Figure 6.

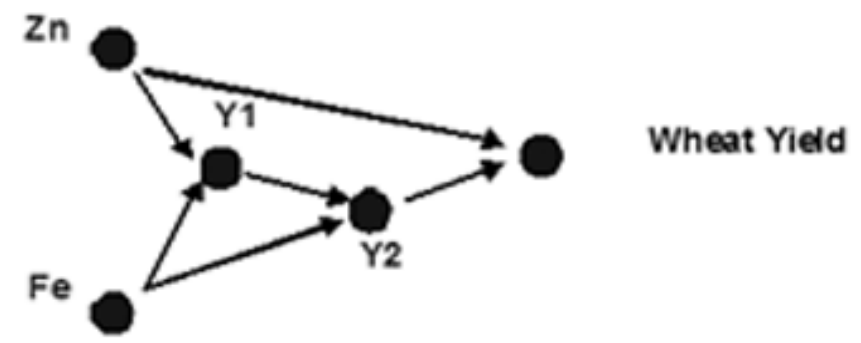

Figure 4. Evolved structure of generalized GMDH neural network.

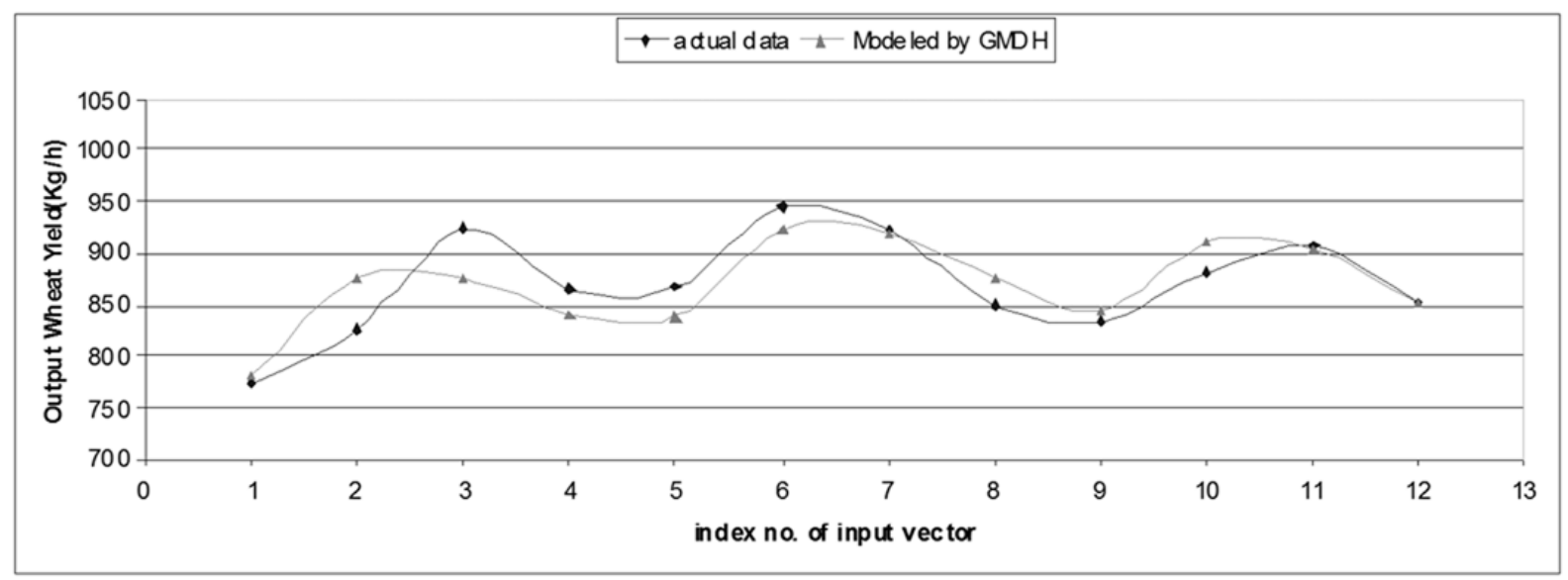

Figure 5. Comparison of actual values and 2-hidden Evolved GMDH neural model. 


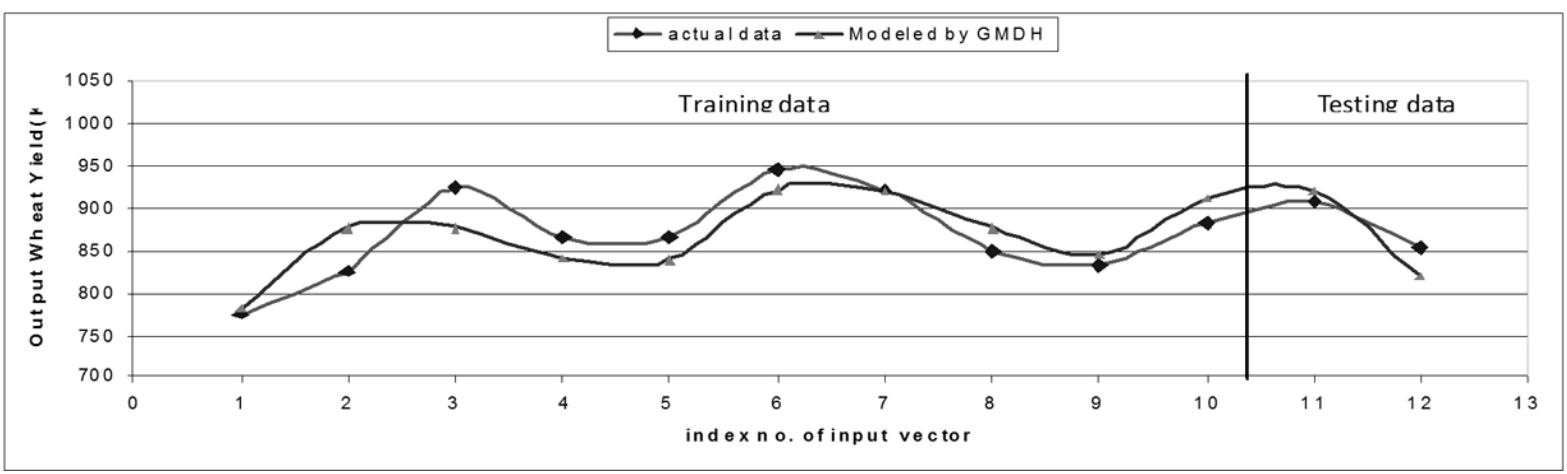

Figure 6. Comparison of actual values and 2-hidden evolved GMDH neural (model1ing \& prediction).

\section{Conclusion}

Evolutionary methods for designing generalized GMDH-type networks have been proposed and successfully used for the modeling and prediction of wheat yield. In this way, a new encoding scheme has been presented to genetically design generalized GMDH-type neural networks in which the connectivity configuration in such networks is not limited to adjacent layers, unlike the conventional GMDH-type neural networks. Such generalization of network's topology provides optimal networks in terms of hidden layers and/or number of neurons and their connectivity configuration so that a polynomial expression for modeling and prediction of the wheat yield can be achieved consequently. Results of this study showed that wheat yield has been affected by the amounts of microelement and this model can be used to predict yield wheat and optimum micro fertilizers mix. The highest yield concerns to fertilizers mix of $\mathrm{Zn}(0.2 \%)$ and Fe (0.5\%), so application of prescribed fertilizer rates can lead to increased yields, and decreased environmental impact of excess fertilizers.

\section{References}

K. J. Astrom, P. Eykhoff. System identification, a survey, Automatica. (1971) 7-123-62.

B. J. Alloway. Zinc in soils and crop nutrition, International zinc Association (IZA). 1 (2004) 128.

S. J. Farlow. Self-organizing method in modeling: GMDH type algorithm. Marcel Dekker Inc.(1984).

S. Ghorbani, B.N. Khiabani, I. Amini, M.R. Ardakani, Effect of iron and zinc on yield and yield components of mutant line's wheat, Asian J. Bio. Sci. 2 (3) (2009) 74-80.

T. Heinzow, R.S.J. Tol, Prediction of crop yields across four climate zones in Germany: An Artificial Neural Network Approach, FNU-34. Hamburg: Centr. Mar. Clim. Res., Hamburg Univ. (2003).

H. Iba, H. deGaris, T. Sato, A numerical approach to genetic programming for system identification. Evolutionary Computation 3(4) (1996) 417-452.

A.G.Ivakhnenko, Polynomial theory of complex systems. IEEE Trans. Syst. Man \& Cybern. SMC-1 (1971) 364-378.

P. Lucca, F. Hurrel, I. Potrykus, Genetic engineering approaches to improve the bioavailability and the level of iron in rice grains. Theor. Appl. Genet, 102,392-397, 2001.

N. Nariman-Zadeh , A. Darvizeh, R. Ahmad-Zadeh, Hybrid genetic design of GMDH-type neural networks using singular value decomposition for modeling and prediction of the explosive cutting Process. J. Eng. Manu. 217 (2003) 779-790.

N. Nariman-zadeh, A. Darvizeh, A. Jamali, A. Moeini. Evolutionary design of generalized polynomial neural networks for modeling and prediction of explosive forming process. J. Mater. Process. Tech. 164(165) (2005) 1561-1571.

W.H. Press, S.A. Teukolsky, W.T. Vetterling, B.P. Flannery, Numerical recipes in FORTRAN. The Art of Scientific Computing (2nd Edition), Cambridge University Press. (1992).

Z. Regal, R.D Graham, Importance of seed Zn content for wheat on zinc deficient soil. Plant Soil, 173 (1995) 259-265.

E. Sanchez, T. Shibata, L.A. Zadeh, Genetic algorithms and fuzzy logic systems. World Scientific, (1997).

X. Yao, Evolving artificial neural networks. Proceedings of IEEE.87 (9) (1999) 1423-1447. 